\title{
On-Demand Intracellular Delivery of Single Particles in Single Cells by 3D Hollow Nanoelectrodes
}

\author{
Jian-An Huang, ${ }^{\dagger}$ Valeria Caprettini, ${ }^{\dagger,+, \S \odot}$ Yingqi Zhao, ${ }^{\dagger}$ Giovanni Melle, $^{\dagger, \star}$ Nicolò Maccaferri, ${ }^{\dagger \odot}$
} Lieselot Deleye, ${ }^{\dagger}$ Xavier Zambrana-Puyalto, ${ }^{\dagger}$ Matteo Ardini,${ }^{\dagger}$ Francesco Tantussi, ${ }^{\dagger \oplus}$ Michele Dipalo, ${ }^{\dagger}$ and Francesco De Angelis, ${ }^{*}+\odot$

${ }^{\dagger}$ Istituto Italiano di Tecnologia, Via Morego 30, 16163 Genova, Italy

${ }^{\ddagger}$ DIBRIS, University of Genoa, Via all’Opera Pia 13, 16145 Genova, Italy

\section{Supporting Information}

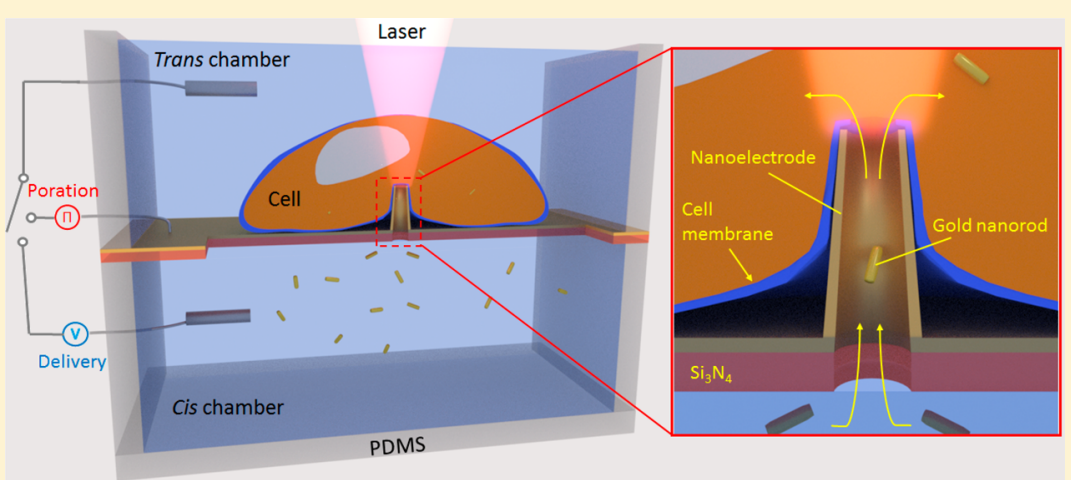

ABSTRACT: Delivery of molecules into intracellular compartments is one of the fundamental requirements in molecular biology. However, the possibility of delivering a precise number of nano-objects with single-particle resolution is still an open challenge. Here we present an electrophoretic platform based on 3D hollow nanoelectrodes to enable delivery of single nanoparticles into single selected cells and monitoring of the single-particle delivery by surface-enhanced Raman scattering (SERS). The gold-coated hollow nanoelectrode capable of confinement and enhancement of electromagnetic fields upon laser illumination can distinguish the SERS signals of a single nanoparticle flowing through the nanoelectrode. Tight wrapping of cell membranes around the nanoelectrodes allows effective membrane electroporation such that single gold nanorods are delivered on demand into a living cell by electrophoresis. The capability of the 3D hollow nanoelectrodes to porate cells and reveal single emitters from the background in continuous flow is promising for the analysis of both intracellular delivery and sampling.

KEYWORDS: Single nanoparticle, intracellular delivery, plasmonics, Raman, SERS, electroporation

Thtracellular delivery of nanoparticles, such as quantum dots, DNA plasmids and gold nanoparticles, is widely used in gene regulation, ${ }^{1}$ disease diagnosis, ${ }^{2}$ drug delivery, and singlecell studies. ${ }^{3-13}$ Nanoparticle endocytosis usually leads to nanoparticle aggregation in endosomal vesicles or nanoparticle attachment to the cell membrane. ${ }^{14}$ However, these vesicles can prevent the trapped nanoparticles from approaching targeted organelles or molecules. ${ }^{15}$ For instance, this has been observed when spherical nucleic acids were used to target specific RNAs. The spherical nucleic acids were found to be trapped in vesicles after endocytosis and thus failed to reflect intracellular RNA transcripts levels. ${ }^{16,17}$ It was reported that only $1-2 \%$ of the endocytosed nanoparticles could escape from the endosomal vesicles into the cytosol. ${ }^{15}$ Additionally, nanoparticle aggregates could distort molecular behavior in mechanistic studies, such as those of intracellular transport by motor proteins. ${ }^{18-24}$ Therefore, physical delivery methods for injecting single particles into the cytoplasm of living cells are highly desired.
Although many different approaches have been developed for cells cultured in vitro, ${ }^{25}$ some important limitations remain. ${ }^{26}$ Among them, the development of quantitative methods has remained difficult. In particular, the possibility of delivering a precise number of nano-objects with singleparticle resolution is an ongoing issue. A recent attempt was laser injection that could deliver single gold nanoparticles into living cells by exciting high surface temperature of the nanoparticles to melt the cytoplasm membrane. The high surface temperature however resulted in low cell viability. ${ }^{27,28}$ Nanochannel electroporation systems focused electric field in the nanochannel to porate cell and deliver cargos with dosage control. $^{29,30}$ Although it demonstrated high cell viability, the

Received: September 17, 2018

Revised: December 21, 2018

Published: January 23, 2019 

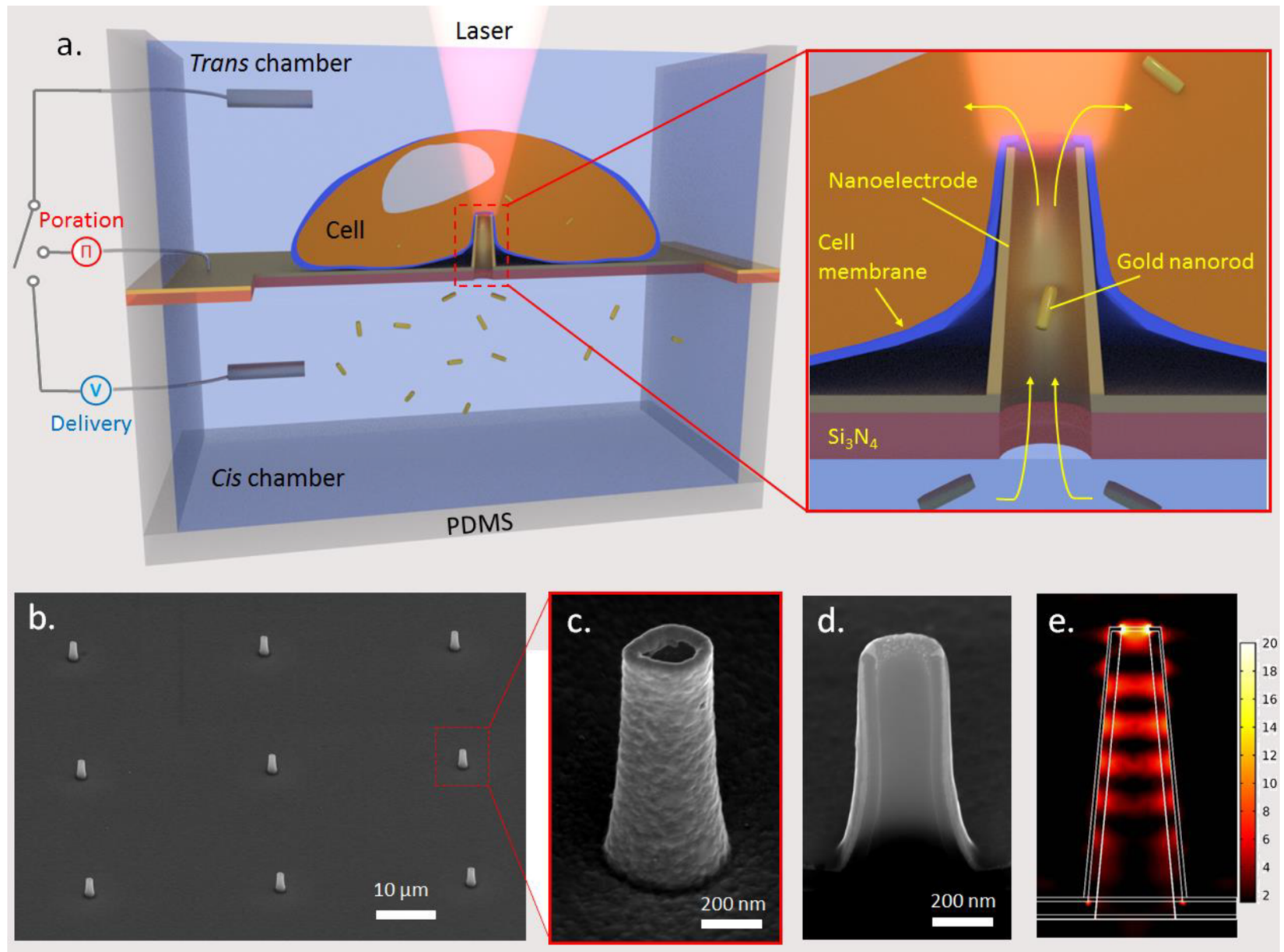

Figure 1. (a) Schematic representation of the 3D hollow nanoelectrode device for single-particle intracellular delivery. The cell is tightly wrapped around the gold-coated hollow nanoelectrode and is first electroporated by a pulsed voltage. Then, the nanorods originally in the cis chamber are delivered into the cell through the hollow nanoelectrode by a DC potential between the two Pt wire electrodes. Inset: a laser beam excites the Raman signals of the delivered nanorods for counting the number of delivered nanorods. SEM images of $3 \mathrm{D}$ hollow nanoelectrode array on $\mathrm{Si}_{3} \mathrm{~N}_{4}$ (b), magnified SEM image of a single nanoelectrode (c), and cross section of the nanoelectrode (d). (e) Simulated electromagnetic field intensity distribution of a nanoelectrode illuminated by linearly polarized plane wave with an enhancement factor between 10 and 20 at the tip.

focused electric field for both cell electroporation and cargo delivery was too high to achieve single-particle delivery.

In recent years, different physical methods based on solidstate nanoneedles ${ }^{31-33}$ and hollow nanotube systems (or nanostraws $)^{34-38}$ have been developed as reliable means of delivery with high cell viability. Still, these methods do not overcome the aforementioned limitations of quantitative intracellular delivery with single-particle resolution. Moreover, delivery solutions able to target single selected cells within a large population would be of additional value due to the growing interest in single-cell analysis.

In this work, we show that single nano-objects can be delivered on demand into cells cultured in vitro by an electrophoretic approach combined with surface-enhanced Raman scattering (SERS), which enables counting of individual nanoparticles in real time and might pave the way to quantitative intracellular delivery with single cell resolution. The method is based on plasmonic hollow nanotubes ${ }^{39}$ that simultaneously act as nanoelectrodes for cell electroporation ${ }^{35}$ and particle delivery (nanochannels) and as plasmonic antennas for Raman signal enhancement. ${ }^{40}$ The concept is represented in Figure 1a. Hollow nanotubes are fabricated on a $\mathrm{Si}_{3} \mathrm{~N}_{4}$ substrate embedded in a polydimethylsiloxane (PDMS) chamber to separate a trans chamber from a cis chamber. The gold-coated hollow nanotube interfacing with the cell membrane acts as a nanoelectrode to generate electropores by a pulsed voltage. Then, a $\mathrm{DC}$ potential is applied to two $\mathrm{Pt}$ wire electrodes in both chambers to deliver nanorods from the cis chamber to the electroporated cell through the hollow nanoelectrodes. In contrast to hollow nanostraws that used only two electrodes for both electroporation and intracellular delivery, our system separates the electroporation by surface electrode from the electrophoretic delivery by two $\mathrm{Pt}$ wire electrode. The separation allowed independent control of the delivery conditions that made single-particle delivery possible. The optical energy can be confined inside the hollow nanotube ${ }^{39}$ such that upon laser illumination, single nanorods entering the nanoelectrodes can be well distinguished from the background in the cis chamber.

Results and Discussion. We first assessed the performance of the $3 \mathrm{D}$ hollow nanoelectrodes by quantifying singleparticle translocation through the hollow nanoelectrodes in phosphate-buffered saline without cells. Raman-tagged gold nanorods $25 \times 90 \mathrm{~nm}^{2}$ in size (Supporting Information Figure S1) were used for electrophoretic translocation. Under $785 \mathrm{~nm}$ laser illumination, single nanorods exhibited stable Raman 

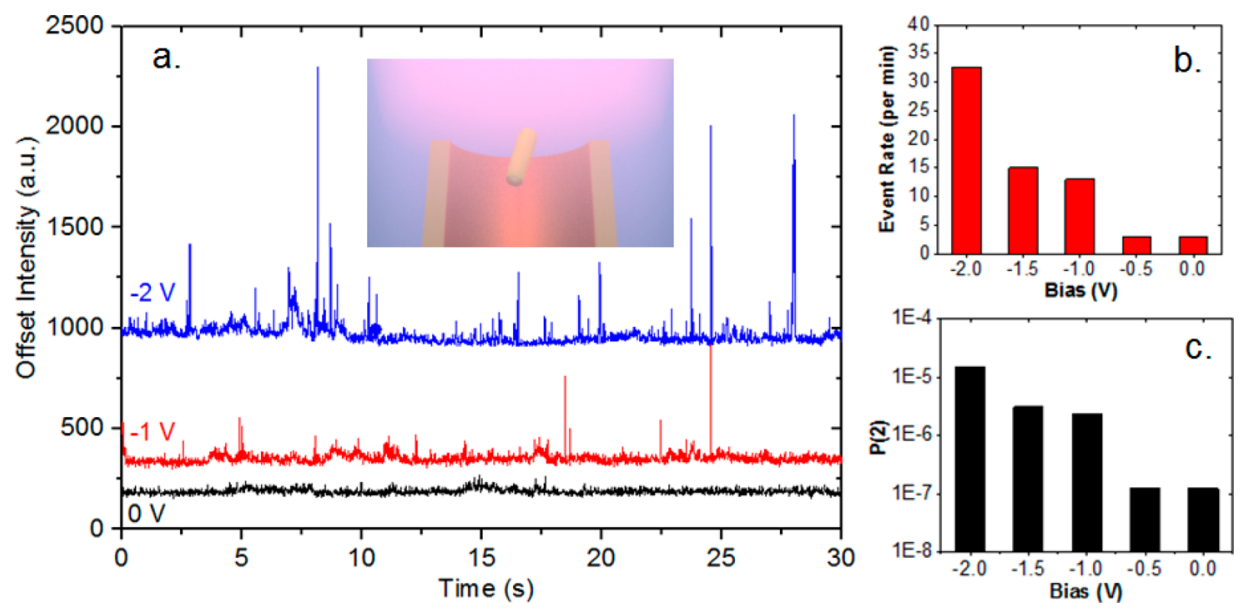

Figure 2. Electrophoretic translocation of nanorods through the hollow nanoelectrodes without cells. (a) Time traces of electrophoretic translocation at a DC bias of $-2 \mathrm{~V}$ (blue curve), $-1 \mathrm{~V}$ (red curve), and $0 \mathrm{~V}$ (black curve, diffusion regime). Inset: schematic of a nanorod passing through the nanoelectrode under laser illumination without cells which corresponds to a burst in the time trace. (b) Measured event rates of nanorod translocation. (c) Probability of the coincidence of 2 nanorods in flow during electrophoretic translocation in the corresponding flow rates in (b) and exposure time $10 \mathrm{~ms}$.

spectra (Figure S2) in which the signal-to-baseline intensity of the Raman band at $593 \mathrm{~cm}^{-1}$ was used as the signal for counting the translocated nanorods.

The hollow nanoelectrodes covered by a $30 \mathrm{~nm}$ thick gold layer had an inner diameter of about $200 \mathrm{~nm}$ and a length of 2 $\mu \mathrm{m}$, as shown in Figure 1c and d. When illuminated with a plane wave at $785 \mathrm{~nm}$, the electromagnetic field intensity was enhanced by a factor between 10 - and 20-fold compared to the intensity of the incident field, as shown in Figure 1e, and as previously demonstrated. ${ }^{39}$

The inner volume of the nanotube was approximately 0.14 $\mathrm{fL}$. However, the experimental detection volume is expected to be even smaller because, as shown in Figure 1e, the plasmonic field is accumulated in a total volume that is smaller than that of the nanotube. The $30 \mathrm{~nm}$-thick gold layer would screen any Raman signals from the cis chamber. By monitoring the Raman signal using an objective with a high numerical aperture (NA = 1) focused at the nanotube tip, we detected only those nanorods that flow through the nanotube. As shown in Figure $2 \mathrm{a}$, a burst with a signal-to-noise intensity ratio no less than 3 in a time trace of the nanorod Raman intensity was considered a translocation event.

Single-Particle Translocation. A critical parameter for ensuring single-particle translocation is the low probability of the coincidence of more than one particle in the nanoelectrode. In the case of nanorod diffusion through the nanoelectrode, the concentration of nanorods dispersed in solution must be carefully adjusted. ${ }^{41}$ We used Poisson statistics for particle diffusion to calculate the optimal concentration (see Methods for details). ${ }^{42}$ According to the calculations, a concentration of $10^{11}$ particles $/ \mathrm{mL}$ leads to 0.014 nanorods on average diffusing inside the hollow nanoelectrode with $0.14 \mathrm{fL}$ volume. The probability of 0,1 , and 2 nanorods diffusing in the hollow nanoelectrode at any time was $0.986,1.38 \times 10^{-2}$, and $9.66 \times 10^{-5}$, respectively. Since the probability of having 2 nanorods passing through the nanochannel simultaneously was very low $\left(<10^{-4}\right)$, we assumed that translocation events at $0 \mathrm{~V}$ (black curves in Figures $2 \mathrm{a}$ and $\mathrm{S} 3 \mathrm{a}$ ) were always single nanorods.

To accelerate the single-particle translocation, DC voltages ranging from -0.5 to $-2 \mathrm{~V}$ were used to drive the negatively charged nanorods. The DC potential was applied between two Pt wire electrodes in the cis and trans chambers separated by a distance of approximately $15 \mathrm{~mm}$. Both the amount and the intensity of the electrophoretic translocation events increased compared to the case of diffusion, as shown in Figure 2a (blue and red curves) and Figure S3a. The burst intensities of the events depend on the distance of the nanorods from the inner sidewall of the nanoelectrode tips during their passage. The nanorods close to the nanoelectrode tip induced plasmonic coupling that led to strong electromagnetic field on the surface of the nanorods and thus high SERS signals (Figure S4).

Raman correlation spectroscopy ${ }^{43,44}$ (see Methods for details) was used to analyze the average number of translocated nanorods and to extract the translocation time. The average numbers of nanorods in all diffusion and biased flow conditions were less than one, proving single-particle translocations (Table S1). Therefore, the number of bursts of the time traces can be counted as event rates of single-particle translocation, which increased with bias voltage in Figure $2 \mathrm{~b}$. The extracted translocation time decreased from $170 \pm 90 \mathrm{~ms}$ for diffusion to $69 \pm 9 \mathrm{~ms}$ at $-2 \mathrm{~V}$ bias (Figure S3b-e). While the translocation time depended on many parameters, such as the nanorod size and the nanoelectrode volume, it is of the same order of magnitude as previously reported single-particle translocation through nanopores. ${ }^{45-48}$

Although the measured event rate increased with the applied potential (Figure $2 \mathrm{~b}$ ), the probability of 2 nanorods flowing through the nanoelectrode simultaneously remained small. Such probability was calculated with the Poisson statistics for particles in flow that considers the irreversible flow of the nanoparticles with a flow rate (or event rate) and an exposure time as the detection time window (see Methods for details). ${ }^{49}$ In the case of $-2 \mathrm{~V}$ biased electrophoretic translocation, the probability of translocating two nanorods simultaneously through the hollow nanoelectrode with $0.14 \mathrm{fL}$ volume at an exposure time of $10 \mathrm{~ms}$ and a measured event rate of 32 per minute was as small as $1.46 \times 10^{-5}$. The probabilities at other bias voltages were even smaller, as shown in Figure $2 c$. Thus, the electrophoretic translocation of a single nanorod was confirmed as the most likely cause for event detection. 
In contrast to particle diffusion, electrophoresis prevents translocated nanorods from returning to the nanoelectrodes. In general, a nanoparticle in the hollow nanoelectrode under bias experiences both electroosmotic force and electrophoretic force. They are proportional to the electric field strength $E$ by zeta potentials of the nanorod $\left(\zeta_{\mathrm{nr}}\right)$ and the nanoelectrode $\left(\zeta_{\mathrm{ne}}\right)$, respectively, that characterize their surface charge. The $\zeta_{\text {ne }}$ is negligible in our experiments conducted in PBS with $\mathrm{pH}$ 7.4 , because the nanoelectrodes were coated with aluminum oxide that has zero charge at $\mathrm{pH} 8 .^{50,51}$ Therefore, the negatively charged nanorod would be mainly driven by the electrophoretic force with an effective velocity toward to the trans chamber as ${ }^{52,53}$

$$
v=\frac{\varepsilon E}{\eta} \zeta_{\mathrm{nr}}
$$

where $\varepsilon$ is the dielectric permittivity of the solution and $\eta$ is the solution viscosity. The event rate depends mainly on the electric field in the nanoelectrode. Therefore, this approach provides an effective method to tune the translocation rate of single nanorods through the nanoelectrode. Here, the electrophoretic voltage of our hollow nanoelectrode system was optimized with the nanorod concentration for efficient intracellular delivery of single nanorods, as shown below.

Intracellular Delivery. To demonstrate intracellular delivery, NIH-3T3 cells were cultured in the trans chamber to allow cell growth on the hollow nanoelectrodes with tight membrane wrapping (Figure 3). In addition to the two Pt wire electrodes
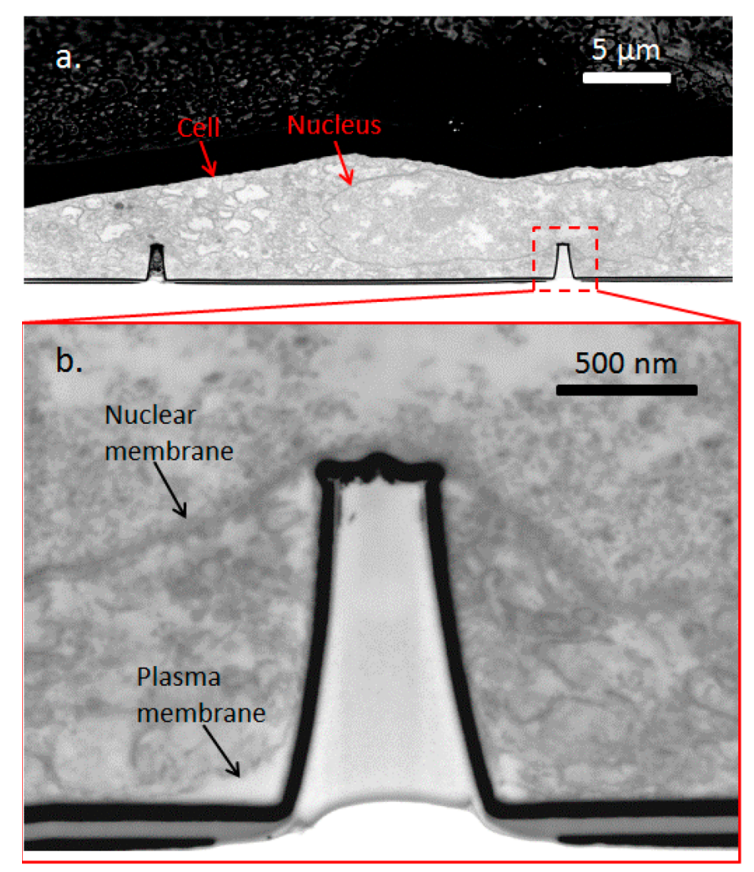

Figure 3. Cross-sectional SEM image of a cell cultured on the nanoelectrodes (a). Magnified SEM image showing that the cell membrane is tightly wrapped around the nanoelectrode (b).

for translocating the nanorods, a cable was connected to the gold layer of the hollow nanoelectrodes for cell membrane electroporation. The membrane was porated by applying a peak-to-peak pulsed voltage of $3 \mathrm{~V}$ for $10 \mathrm{~s}$ with pulse length of $100 \mu \mathrm{s}$ and a frequency of $20 \mathrm{~Hz}$ between the Pt wire electrode in Phosphate Buffered Saline (PBS) in the trans chamber and the hollow nanoelectrodes. After the electropores were generated in the cell membrane, electrophoretic delivery of the nanorods was conducted with DC voltage $(-1$ to $-2 \mathrm{~V})$ between the two $\mathrm{Pt}$ wire electrodes in the trans and cis chambers. Gold nanorods with $10 \times 40 \mathrm{~nm}$ in size were used to facilitate delivery, because the $100 \mu$ s pulse were expected to generate small electropores. ${ }^{54}$ Intracellular deliveries of the nanorods through the nanoelectrodes were monitored in time traces of the nanorod Raman intensities before and after electroporation as described in the previous section. Subsequently, Raman mappings on the cells laying on the nanoelectrodes were performed to check the distribution of the delivered nanorods.

The single-particle delivery proved to be possible only by the $-2 \mathrm{~V}$ bias. As shown in a typical time trace with baseline close to zero in Figure 4a, no bursts were observed under the electrophoretic bias from -1 to $-1.5 \mathrm{~V}$ after the electroporation. The first delivery event emerged about $30 \mathrm{~s}$ after the trigger of the $-2 \mathrm{~V}$ bias. Once the $-2 \mathrm{~V}$ bias was turned off, no events appeared again until another electroporation and $-2 \mathrm{~V}$ bias applied.

The distributions of the delivered nanorods as red dots in optical bright-field images (Figure $4 c-h$ ) confirmed successful single-particle intracellular delivery. The red dots represent the signal-to-baseline Raman intensity of the nanorods, and move within the cell during $15 \mathrm{~min}$ from the end of the delivery. In previous reports on the endocytic uptake of nanoparticles, the Raman signals of intracellular nanoparticles were colocalized with black dots in bright-field optical images that corresponded to intravesicular nanoparticle aggregates. ${ }^{55,56}$ Our case is in strong contrast with these reports because no black dots were observed in the bright-field images overlapping the red dots. Since it takes at least $2 \mathrm{~h}$ for motor proteins to capture and aggregate single intracellular nanoparticles, ${ }^{57,58}$ we ascribed the red dots to single nanorods, which could not be resolved by the bright-field $60 \times$ objective.

The electroporation and $-2 \mathrm{~V}$ bias electrophoresis had little effect to cell viability (Figure S5). Similar single-particle images reproducibly appeared in successful deliveries by $-2 \mathrm{~V}$ bias with high cell viability, such as in Figure S6. Therefore, the -2 $\mathrm{V}$ electrophoretic bias provided a switch to trigger the singleparticle delivery.

Two possible explanations might clarify the unsuccessful nanorod delivery at bias $<-2 \mathrm{~V}$. The first one was the high membrane resistance of approximately $14 \mathrm{M} \Omega$, as shown in both membrane 1 and membrane 2 of one intact cell in an equivalent circuit in Figure 5a,b. ${ }^{29}$ Cell interior was treated as conductive medium because its resistance was negligible compared to the membrane resistance. The resistance of a bare nanoelectrode was calculated as $27 \mathrm{M} \Omega$ by $R=4 \mathrm{~L} /$ $\left(\pi d^{2} \sigma_{\mathrm{PBS}}\right)$ where $L$ is the length and $d$ is the inner diameter of the nanoelectrode, and $\sigma_{\mathrm{PBS}}=0.8 \mathrm{~S} / \mathrm{m}$ is the conductivity of PBS. $^{29}$ Thus, even if the membrane 1 that wrapped the nanoelectrode was porated and became conductive, the membrane 2 from the same cell still presented a high resistance of $14 \mathrm{M} \Omega$, which caused a reduction of the effective applied potential for delivery $\left(V=V_{\mathrm{m} 1}+V_{\mathrm{m} 2}+V_{\mathrm{ne}}\right)$. Moreover, the transient electropores continuously shrink after electroporation, ${ }^{59,60}$ leading to a total membrane resistance between 14 to $28 \mathrm{M} \Omega$.

Second, the intracellular contents that leaked from the porated cell into the nanoelectrode might resist the inward nanorod movement. We have observed DNA leaked into nanoelectrodes from porated cells in another report of 

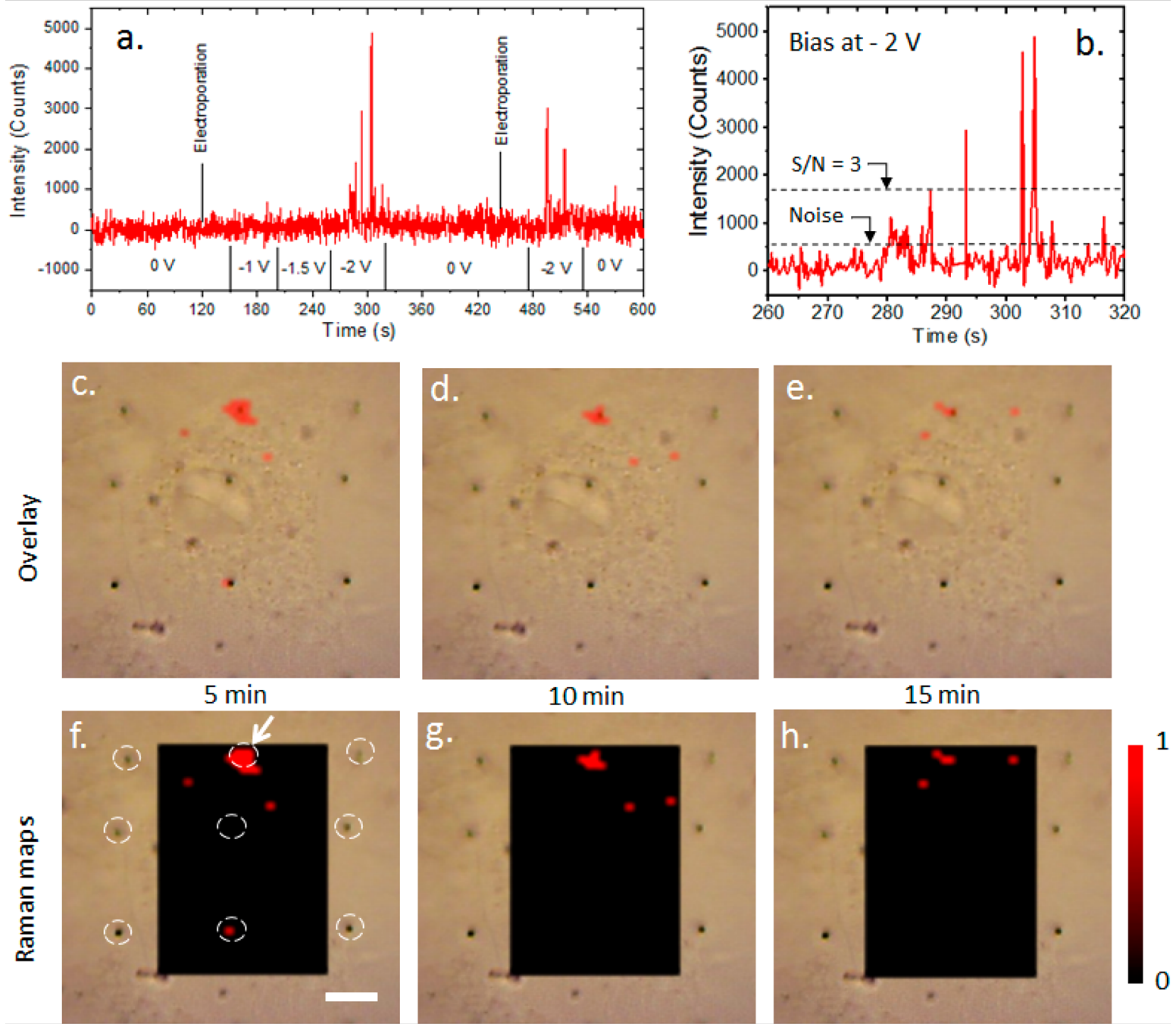

Figure 4. (a) Time trace of the electrophoretic intracellular delivery of nanorods at a bias of $0,-1,-1.5$, and $-2 \mathrm{~V}$ before and after electroporation. (b) Magnified time trace of intracellular delivery of nanorods at $-2 \mathrm{~V}$ bias extracted from (a); bursts with signal-to-noise (S/N) ratio $>3$ are regarded as delivery events. Bright-field images of the cell overlaid with corresponding Raman maps (f, g, h) of the delivered nanorods at 5 min (c, f), $10 \mathrm{~min}(\mathrm{~d}, \mathrm{~g})$, and $15 \mathrm{~min}(\mathrm{e}, \mathrm{h})$ after the end of the time trace in (a). In (f), white dotted circles are the positions of the nanoelectrodes, while the nanoelectrode marked by the white arrow was the delivering nanoelectrode that was monitored by the time trace in (a). The white scale bar is $10 \mu \mathrm{m}$, and the red color bar representing normalized Raman intensity applies to all images.

nanoelectrodes electroporation. ${ }^{40}$ In fact, intracellular sampling by hollow nanoneedles with $150-450 \mathrm{~nm}$ diameters have also demonstrated that $2-5 \%$ of the proteins and small molecules would diffuse from a cell through the nanoneedle within 5-10 min after the cell membrane was electroporated. ${ }^{61,62}$ The cytosolic contents might come out even faster in our cases, because positively charged molecules, such as some amino acids, ${ }^{63}$ could be dragged out by the applied biases.

Furthermore, nanorod clogging in some nanoelectrodes also suggested cytosolic content leakage into nanoelectrodes. In the overlay images (Figure $4 c-e$ ), the nanoelectrode that had delivered the nanorods continuously overlapped with red dots in the Raman maps indicated that the nanorods were accumulated inside. A cross-sectional scanning electron microscopy (SEM) image of fixed cells on such a nanoelectrode in Figure 5c shows nanorods clogged inside that caused the signal accumulation. In particular, the cell membrane remained tightly wrapped on the nanoelectrode even after the electroporation and delivery, such that the nanorod clogging was only due to intracellular molecules. For example, amino acids adsorbed on the nanorods would reduce surface charges on the nanorods and result in nanorod aggregation. ${ }^{64-67}$ Besides, the clogged nanorods could be moved back into the cis chamber by applying a positive DC bias. The fluctuating time trace baseline of the clogged nanoelectrode decreased with positive biases to near zero as shown in Figure S7. This reversible nanorod movement through the nanoelectrodes could be a way for controllable intracellular sampling.

The number of the events in the time trace should be equal to the number of single nanorods delivered in the cell by the monitored nanoelectrode. However, we could not prove such correspondence in the Raman maps because one cell was electroporated and delivered by more than one nanoelectrode simultaneously. Quantitative single-particle delivery by single nanoelectrode electroporation and delivery at single cell can be realized by integrating microelectrode array technology to address only one nanoelectrode among the array, ${ }^{40,68}$ or simply by fabricating just one nanoelectrode per chip. On the other hand, our Raman mapping was performed spot-by-spot by 

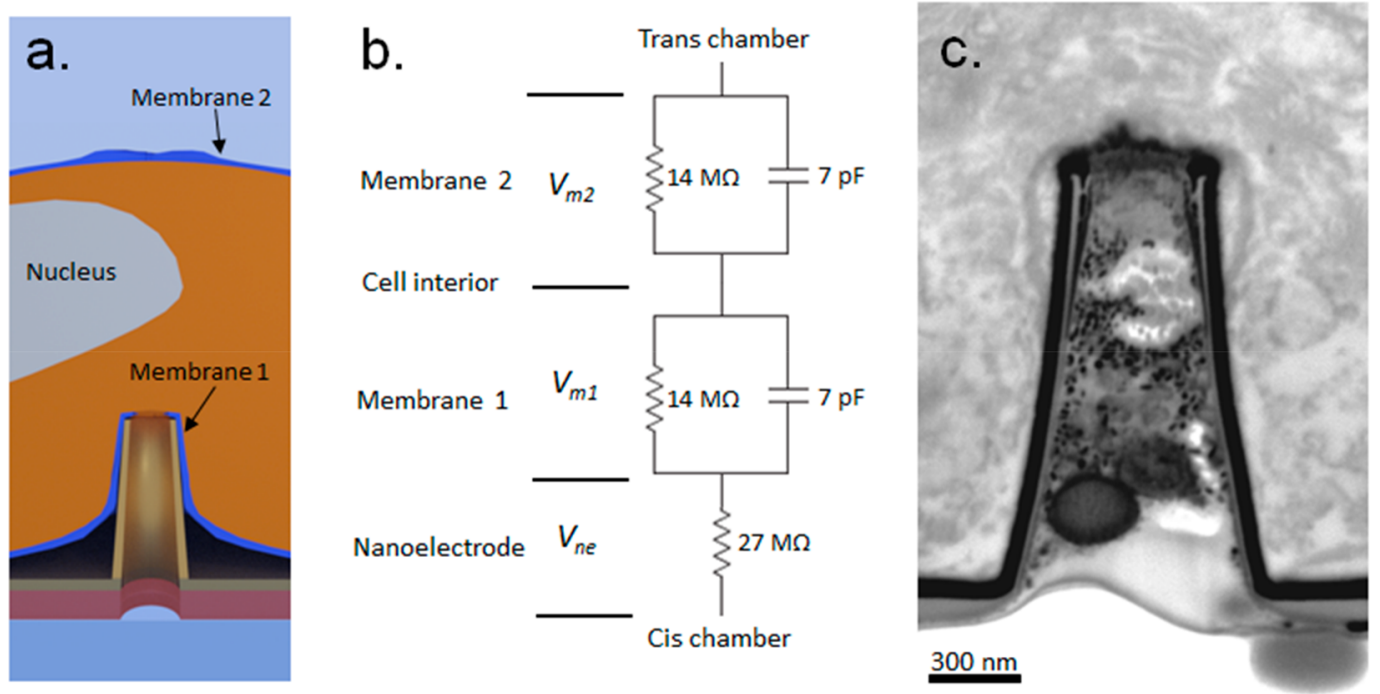

Figure 5. (a) Schematic representation of a cell grown on the nanoelectrode.(b) Electrical equivalent circuit of a intact cell grown on the nanoelectrode. (c) Cross-sectional SEM image of the delivering nanoelectrode in Figure $4 \mathrm{f}$ which was clogged with gold nanorods (black dots).

mechanically moving the sample stage. As each spot required $100 \mathrm{~ms}$ to map, the whole mapping process was usually completed in 3-10 min depending on the cell sizes, which was too slow to trace the real-time distribution of the nanorods. Nevertheless, the temporal limitation due to the stage-scanning Raman microscope can be readily overcome using a laserscanning Raman microscope. ${ }^{69,70}$

In conclusion, we demonstrated the electrophoretic intracellular delivery of nanorods with the capability of triggering and detecting single events, thus providing a method for accurate quantitative delivery. The platform is based on multifunctional plasmonic hollow nanotubes that can work as (i) nanoelectrodes for cell electroporation, (ii) nanochannels for electrophoretic delivery, and (iii) plasmonic antennas to enhance the optical signals of nano-objects translocating through the channel. The tight wrapping of the cell membrane around the nanoelectrodes allowed for the generation of electropores which were large enough to allow the passage of nanorods while still preserving the membrane adhesion after electroporation and thereby preventing nanorod leakage.

The advantage of the gold-coated nanoelectrode is that it can screen the background of the nanoparticle reservoir in the cis chamber and enhance the signal of the nanoparticle that passes through the nanoelectrode. In this work, we used SERS signals of gold nanorods to map the cell for locating delivered nanorods easily by avoiding cell autofluoresence. Our platform can be extended to delivering other nano-objects, such as fluorescent nanoparticles, by minor adjustment to the nanoelectrode configuration, for example, deposition of thicker gold layer to screen fluorescence. By a simple refinement of the platform, it is possible to realize one nanoelectrode per cell for the discrimination of cells that have and have not received a nano-object, which would be ideal for use in the emerging field of single-cell analysis. Finally, cells remained alive after the delivery on our platform and could be processed for other applications and analysis. As hollow nanotubes have been demonstrated to extract cytosolic content from living cells, ${ }^{61}$ in the future, such a platform could be used for the real-time monitoring and on-chip analysis of the intracellular content, including proteins, DNA and miRNA. ${ }^{71}$
Methods. Materials. Raman-tagged gold nanorods dispersed in deionized water were purchased from Nanopartz Inc. (Loveland, CO) with Nile blue A (NBA) as the Raman reporter and stabilized by carboxyl groups. The gold nanorods were either $10 \times 40 \mathrm{~nm}^{2}$ or $25 \times 90 \mathrm{~nm}^{2}$ in size, with transverse plasmonic resonance at a wavelength of $510 \mathrm{~nm}$ and longitudinal plasmonic resonance at a wavelength of $780 \mathrm{~nm}$. The zeta potential at $\mathrm{pH}=7.4$ and the concentration were -18 $\mathrm{mV}$ and $4 \times 10^{13}$ particles $/ \mathrm{mL}$, respectively, and those of the $25 \times 90 \mathrm{~nm}^{2}$ nanorods were $-15 \mathrm{mV}$ and $4 \times 10^{12}$ particles/ $\mathrm{mL}$, respectively.

Device Fabrication. To fabricate the 3D hollow nanoelectrodes, S1813 photoresist (Shipley) was spin-coated on a 1 $\times 1 \mathrm{~cm}^{2} \mathrm{Si}_{3} \mathrm{~N}_{4}$ membrane at $4000 \mathrm{rpm}$ for $1 \mathrm{~min}$ and soft baked at $95{ }^{\circ} \mathrm{C}$ for $5 \mathrm{~min}$. After sputtering a $7 \mathrm{~nm}$ thick titanium and a $20 \mathrm{~nm}$ thick gold layer on the back of the $\mathrm{Si}_{3} \mathrm{~N}_{4}$ membrane, focused ion beam milling (FIB, FEI Helios NanoLab 650 DualBeam) at a voltage of $30 \mathrm{keV}$ and a current from 0.23 to $2.5 \mathrm{nA}$ was used to drill hole arrays in the back of the $\mathrm{Ti} / \mathrm{Au}$-coated $\mathrm{Si}_{3} \mathrm{~N}_{4}$ sample. Different FIB currents correspond to different nanotube inner diameters. Then, the sample was ashed by oxygen plasma at $100 \mathrm{~W}$ for $2 \mathrm{~min}$ to smooth the photoresist and was then developed in acetone for 2 min to form polymer nanotube arrays. Then, the nanotube arrays were thinned down by oxygen plasma at $100 \mathrm{~W}$ for 2 min. An alumina layer of $5 \mathrm{~nm}$ was deposited on the back of the sample by atomic layer deposition (Oxford Instruments) to neutralize the surface charge. After being coated with a $7 \mathrm{~nm}$ thick Ti layer and a $30 \mathrm{~nm}$ thick gold layer by sputtering at a $45^{\circ}$ tilt angle with rotation to ensure uniform coating, the sample was annealed on a hot plate at $200{ }^{\circ} \mathrm{C}$ in the air for $1 \mathrm{~h}$ and allowed to cool naturally. The as-made nanoelectrodes were attached with a cable by silver paste and embedded in a microfluidic chamber made from polydimethylsiloxane (PDMS, Dow Corning SYLGARD 184 silicone elastomer) at $60{ }^{\circ} \mathrm{C}$ for approximately $40 \mathrm{~min}$.

Cell Culture. We used NIH-3T3 cells for the delivery experiments. Before seeding the cells, the devices were irradiated with UV rays for $20 \mathrm{~min}$ in a laminar-flow hood to sterilize them. The devices were treated overnight with complete DMEM, containing $1 \%$ pen/strep antibiotic and 
$10 \%$ fetal bovine serum (Sigma-Aldrich), to saturate the PDMS chamber. Then, NIH-3T3 cells were seeded on the devices at a concentration of $0.7 \times 10^{4}$ cells $/ \mathrm{cm}^{2}$ and incubated at $37{ }^{\circ} \mathrm{C}$ in a $5 \% \mathrm{CO}_{2}$ atmosphere for $24 \mathrm{~h}$ in complete DMEM before the experiments were performed.

FIB Cross Section and SEM Imaging of Cells. After Raman measurement, cells were washed three times with PBS and fixed with glutaraldehyde solution $(2.5 \%)$ in $\mathrm{Na}$ cacodylate buffer $(0.1 \mathrm{M})$ overnight at $4{ }^{\circ} \mathrm{C}$ for FIB cross section. A dualbeam Helios Nanolab 650 (Thermo Fisher Scientific) microscope was used to cut trenches in the fixed cells for the cross sections. Cells were treated with a heavy metal staining protocol discussed elsewhere. ${ }^{72}$ High ionic current (9.3 or $0.79 \mathrm{nA}$ ) was used to slice the specimens after Pt deposition by the gas injection system of the instrument. SEM imaging was performed with the samples tilted at $52^{\circ}$ with respect to the electron beam, and backscattered electrons were collected using a TLD detector in immersion mode. The acceleration of the primary electrons was $3 \mathrm{kV}$, and the current used in the imaging process was $i=0.40 \mathrm{nA}$. The crosssectioned images are presented with inverted colors to emphasize the cellular membranes.

Raman Measurements. Raman measurements were obtained by a Renishaw inVia Raman spectrometer with a Nikon $60 \times$ water immersion objective with a 1.0 NA delivering a $785 \mathrm{~nm}$ laser with a power of approximately $3.27 \mathrm{~mW}$. Intracellular nanoparticle delivery was measured using an Andor EMCCD camera (DU970P-BVF) integrated into the Renishaw spectrometer with an exposure time of $10 \mathrm{~ms}$. Cell mapping was conducted with the Renishaw CCD camera at an exposure time of $300 \mathrm{~ms}$ and a step of $1 \mu \mathrm{m}$.

Poisson Statistics for Particle Diffusion. When an average of $\langle N\rangle$ nanoparticles are diffusing in a given volume, the probability of having $\mathrm{m}$ nanoparticles at any time in the volume can be calculated by the Poisson statistics for diffusion: ${ }^{42}$

$$
P(m)=\frac{\langle N\rangle^{m}}{m !} \exp (-\langle N\rangle)
$$

Poisson Statistics for Particles in Flow. When nanoparticles are in flow in a given volume at a rate of $c$, the probability of having $n$ nanoparticles in the volume at time $\Delta t$ can be calculated by the Poisson statistics in flow: ${ }^{49}$

$$
P(n)=\frac{(c \Delta t)^{n}}{n !} \exp (-c \Delta t)
$$

Raman Correlation Spectroscopy. Time traces of Raman signals, such as those in Figure S3a, were used to compute an autocorrelation function of the kind $G(\tau)=\langle F(t) F(t+\tau)\rangle /$ $\langle F(t)\rangle^{2}$, where $F(t)$ is the Raman time trace, $\tau$ is the lag time, and \langle\rangle indicates time averaging. Average number of translocated nanorods and the diffusion/translocation time in Table S1 were extracted by fitting the autocorrelation function with a 2D Gaussian diffusion model: ${ }^{73}$

$$
G(\tau)=1+\frac{\gamma}{N\left(1+\frac{\tau}{\tau_{\mathrm{D}}}\right)}
$$

where $\gamma$ is the Gaussian point source function-model dependent gamma factor, $N$ is the average number of particles in the detection volume, and $\tau_{\mathrm{D}}$ is the average diffusion time of the particles through the detection volume.
Electromagnetic Simulations. Numerical simulations based on the Finite-Element Method implemented in the RF Module of Comsol Multiphysics were carried out to investigate the electromagnetic response of hollow nanoelectrode. The dimensions of the simulated structure were set according to the average size obtained from SEM images. A dielectric constant $n=1.33$ was used for water, and $n=1.5$ was set for the glass substrate. The refractive index of $\mathrm{Au}$ was taken from Rakic et al. ${ }^{74}$ The model computes the electromagnetic field in each point of the simulation region. The unit cell was set to be $1500 \mathrm{~nm}$ wide in both $x$ - and $y$-directions and $2000 \mathrm{~nm}$ along the $z$-direction, with perfect matching layers ( $200 \mathrm{~nm}$ thick) at the borders. A linearly polarized collimated beam is impinging on the structure from the top side.

\section{ASSOCIATED CONTENT}

\section{S Supporting Information}

The Supporting Information is available free of charge on the ACS Publications website at DOI: 10.1021/acs.nanolett.8b03764.

SEM images and Raman spectra of the gold nanorods; Time traces and Raman correlation spectroscopy of the biased nanorod translocations; Electromagnetic field simulation of the nanorod at the nanoelectrode tip; Cell viability test; More example of intracellular electrophoretic delivery; Time trace of nanorods clogged at the nanoelectrode (PDF)

\section{AUTHOR INFORMATION}

\section{Corresponding Author}

*E-mail: francesco.deangelis@iit.it.

ORCID

Valeria Caprettini: 0000-0002-2743-5536

Nicolò Maccaferri: 0000-0002-0143-1510

Francesco Tantussi: 0000-0002-0812-082X

Michele Dipalo: 0000-0002-1823-8231

Francesco De Angelis: 0000-0001-6053-2488

Present Address

${ }^{\S}$ V.C.: Centre For Craniofacial \& Regenerative Biology, King's College London, London, UK. N.M.: Physics and Materials Science Research Unit, University of Luxembourg, L-1511, Luxembourg

\section{Author Contributions}

F.D.A. conceived and supervised the work. J.A.H. and M.A. prepared the nanoparticles. J.A.H., V.C. and Y.Z. designed and fabricated the devices. V.C., G.M. and L.D. cultured the cells. J.A.H., V.C., F.T. and M.D. designed the optical-electrical setup. J.A.H. performed the Raman measurements. V.C. did the FIB cross-section and SEM imaging. J.A.H. and Y.Z. analyzed the data. X.Z.P. did the Raman correlation spectroscopy analysis. N.M. performed the electromagnetic simulations. All authors contributed to the manuscript preparation.

\section{Notes}

The authors declare no competing financial interest.

\section{ACKNOWLEDGMENTS}

We thank Dr. Rosario Capozza for valuable discussions, and Dr. Giorgia Giovannini for analyzing nanorod stability. The research leading to these results was funded by the European Research Council under the European Union's Seventh 
Framework Programme (FP/2007-2013)/ERC Grant Agreement no. [616213] and CoG: Neuro-Plasmonics.

\section{REFERENCES}

(1) Xia, Y.; Zhang, R.; Wang, Z.; Tian, J.; Chen, X. Recent advances in high-performance fluorescent and bioluminescent RNA imaging probes. Chem. Soc. Rev. 2017, 46 (10), 2824-2843.

(2) Chinen, A. B.; Guan, C. M.; Ferrer, J. R.; Barnaby, S. N.; Merkel, T. J.; Mirkin, C. A. Nanoparticle Probes for the Detection of Cancer Biomarkers, Cells, and Tissues by Fluorescence. Chem. Rev. 2015, 115 (19), 10530-10574.

(3) Doane, T. L.; Burda, C. The unique role of nanoparticles in nanomedicine: imaging, drug delivery and therapy. Chem. Soc. Rev. 2012, 41 (7), 2885-2911.

(4) Kang, B.; Afifi, M. M.; Austin, L. A.; El-Sayed, M. A. Exploiting the Nanoparticle Plasmon Effect: Observing Drug Delivery Dynamics in Single Cells via Raman/Fluorescence Imaging Spectroscopy. ACS Nano 2013, 7 (8), 7420-7427.

(5) Zheng, X. T.; Li, C. M. Single cell analysis at the nanoscale. Chem. Soc. Rev. 2012, 41 (6), 2061-2071.

(6) Zhong, Y.; Peng, F.; Bao, F.; Wang, S.; Ji, X.; Yang, L.; Su, Y.; Lee, S.-T.; He, Y. Large-Scale Aqueous Synthesis of Fluorescent and Biocompatible Silicon Nanoparticles and Their Use as Highly Photostable Biological Probes. J. Am. Chem. Soc. 2013, 135 (22), $8350-8356$

(7) He, Y.; Kang, Z.-H.; Li, Q.-S.; Tsang, C. H. A.; Fan, C.-H.; Lee, S.-T. Ultrastable, Highly Fluorescent, and Water-Dispersed SiliconBased Nanospheres as Cellular Probes. Angew. Chem., Int. Ed. 2009, 48 (1), 128-132.

(8) Zimmerman, J. F.; Parameswaran, R.; Murray, G.; Wang, Y.; Burke, M.; Tian, B. Cellular uptake and dynamics of unlabeled freestanding silicon nanowires. Sci. Adv. 2016, 2 (12), e1601039.

(9) Leijten, J.; Khademhosseini, A. From Nano to Macro: Multiscale Materials for Improved Stem Cell Culturing and Analysis. Cell Stem Cell 2016, 18 (1), 20-24.

(10) Xi, W.; Schmidt, C. K.; Sanchez, S.; Gracias, D. H.; CarazoSalas, R. E.; Jackson, S. P.; Schmidt, O. G. Rolled-up Functionalized Nanomembranes as Three-Dimensional Cavities for Single Cell Studies. Nano Lett. 2014, 14 (8), 4197-4204.

(11) Hartmann, R.; Weidenbach, M.; Neubauer, M.; Fery, A.; Parak, W. J. Stiffness-Dependent In Vitro Uptake and Lysosomal Acidification of Colloidal Particles. Angew. Chem., Int. Ed. 2015, 54 (4), $1365-1368$.

(12) Chen, X.; Zhang, W. Diamond nanostructures for drug delivery, bioimaging, and biosensing. Chem. Soc. Rev. 2017, 46 (3), 734-760.

(13) Kurz, V.; Tanaka, T.; Timp, G. Single Cell Transfection with Single Molecule Resolution Using a Synthetic Nanopore. Nano Lett. 2014, 14 (2), 604-611.

(14) Chou, L. Y. T.; Ming, K.; Chan, W. C. W. Strategies for the intracellular delivery of nanoparticles. Chem. Soc. Rev. 2011, 40 (1), 233-245.

(15) Gilleron, J.; Querbes, W.; Zeigerer, A.; Borodovsky, A.; Marsico, G.; Schubert, U.; Manygoats, K.; Seifert, S.; Andree, C.; Stoeter, M.; Epstein-Barash, H.; Zhang, L.; Koteliansky, V.; Fitzgerald, K.; Fava, E.; Bickle, M.; Kalaidzidis, Y.; Akinc, A.; Maier, M.; Zerial, M. Image-based analysis of lipid nanoparticle-mediated siRNA delivery, intracellular trafficking and endosomal escape. Nat. Biotechnol. 2013, 31 (7), 638-U102.

(16) Budik, S.; Tschulenk, W.; Kummer, S.; Walter, I.; Aurich, C. Evaluation of SmartFlare probe applicability for verification of RNAs in early equine conceptuses, equine dermal fibroblast cells and trophoblastic vesicles. Reprod., Fertil. Dev. 2017, 29 (11), 2157-2167.

(17) Czarnek, M.; Bereta, J. SmartFlares fail to reflect their target transcripts levels. Sci. Rep. 2017, 7, 11682.

(18) Cui, B. X.; Wu, C. B.; Chen, L.; Ramirez, A.; Bearer, E. L.; Li, W. P.; Mobley, W. C.; Chu, S. One at a time, live tracking of NGF axonal transport using quantum dots. Proc. Natl. Acad. Sci. U. S. A. 2007, 104 (34), 13666-13671.
(19) Zhang, K.; Osakada, Y.; Vrljic, M.; Chen, L. A.; Mudrakola, H. V.; Cui, B. X. Single-molecule imaging of NGF axonal transport in microfluidic devices. Lab Chip 2010, 10 (19), 2566-2573.

(20) Courty, S.; Luccardini, C.; Bellaiche, Y.; Cappello, G.; Dahan, M. Tracking individual kinesin motors in living cells using single quantum-dot imaging. Nano Lett. 2006, 6 (7), 1491-1495.

(21) Pinaud, F.; Clarke, S.; Sittner, A.; Dahan, M. Probing cellular events, one quantum dot at a time. Nat. Methods 2010, 7 (4), 275285.

(22) Leduc, C.; Si, S.; Gautier, J. J.; Gao, Z. H.; Shibu, E. S.; Gautreau, A.; Giannone, G.; Cognet, L.; Lounis, B., Single-molecule imaging in live cell using gold nanoparticles. In Biophysical Methods in Cell Biology, Paluch, E. K., Ed.; 2015; Vol. 125, pp 13-27.

(23) Cognet, L.; Tardin, C.; Boyer, D.; Choquet, D.; Tamarat, P.; Lounis, B. Single metallic nanoparticle imaging for protein detection in cells. Proc. Natl. Acad. Sci. U. S. A. 2003, 100 (20), 11350-11355.

(24) Kang, J. W.; So, P. T. C.; Dasari, R. R.; Lim, D. K. High Resolution Live Cell Raman Imaging Using Subcellular OrganelleTargeting SERS-Sensitive Gold Nanoparticles with Highly Narrow Intra-Nanogap. Nano Lett. 2015, 15 (3), 1766-1772.

(25) Yan, L.; Zhang, J. F.; Lee, C. S.; Chen, X. F. Micro- and Nanotechnologies for Intracellular Delivery. Small 2014, 10 (22), 4487-4504.

(26) Stewart, M. P.; Sharei, A.; Ding, X. Y.; Sahay, G.; Langer, R.; Jensen, K. F. In vitro and ex vivo strategies for intracellular delivery. Nature 2016, 538 (7624), 183-192.

(27) Li, M.; Lohmuller, T.; Feldmann, J. Optical Injection of Gold Nanoparticles into Living Cells. Nano Lett. 2015, 15 (1), 770-775.

(28) Zhong, J.; Liu, H. J.; Maruyama, H.; Masuda, T.; Arai, F. Continuous-wave laser-assisted injection of single magnetic nanobeads into living cells. Sens. Actuators, B 2016, 230, 298-305.

(29) Boukany, P. E.; Morss, A.; Liao, W.-c.; Henslee, B.; Jung, H.; Zhang, X.; Yu, B.; Wang, X.; Wu, Y.; Li, L.; Gao, K.; Hu, X.; Zhao, X.; Hemminger, O.; Lu, W.; Lafyatis, G. P.; Lee, L. J. Nanochannel electroporation delivers precise amounts of biomolecules into living cells. Nat. Nanotechnol. 2011, 6 (11), 747-754.

(30) Chang, L. Q.; Bertani, P.; Gallego-Perez, D.; Yang, Z. G.; Chen, F.; Chiang, C. L.; Malkoc, V.; Kuang, T. R.; Gao, K. L.; Lee, L. J.; Lu, W. 3D nanochannel electroporation for high-throughput cell transfection with high uniformity and dosage control. Nanoscale 2016, 8 (1), 243-252.

(31) Shalek, A. K.; Robinson, J. T.; Karp, E. S.; Lee, J. S.; Ahn, D.-R.; Yoon, M.-H.; Sutton, A.; Jorgolli, M.; Gertner, R. S.; Gujral, T. S.; MacBeath, G.; Yang, E. G.; Park, H. Vertical silicon nanowires as a universal platform for delivering biomolecules into living cells. Proc. Natl. Acad. Sci. U. S. A. 2010, 107 (5), 1870-1875.

(32) Chiappini, C.; Martinez, J. O.; De Rosa, E.; Almeida, C. S.; Tasciotti, E.; Stevens, M. M. Biodegradable Nanoneedles for Localized Delivery of Nanoparticles in Vivo: Exploring the Biointerface. ACS Nano 2015, 9 (5), 5500-5509.

(33) Wang, Y.; Yang, Y.; Yan, L.; Kwok, S. Y.; Li, W.; Wang, Z.; Zhu, X.; Zhu, G.; Zhang, W.; Chen, X.; Shi, P. Poking cells for efficient vector-free intracellular delivery. Nat. Commun. 2014, 5, 4466.

(34) Messina, G. C.; Dipalo, M.; La Rocca, R.; Zilio, P.; Caprettini, V.; Zaccaria, R. P.; Toma, A.; Tantussi, F.; Berdondini, L.; De Angelis, F. Spatially, Temporally, and Quantitatively Controlled Delivery of Broad Range of Molecules into Selected Cells through Plasmonic Nanotubes. Adv. Mater. 2015, 27 (44), 7145.

(35) Caprettini, V.; Cerea, A.; Melle, G.; Lovato, L.; Capozza, R.; Huang, J.-A.; Tantussi, F.; Dipalo, M.; De Angelis, F. Soft electroporation for delivering molecules into tightly adherent mammalian cells through 3D hollow nanoelectrodes. Sci. Rep. 2017, 7,8524

(36) Xie, X.; Xu, A. M.; Leal-Ortiz, S.; Cao, Y. H.; Garner, C. C.; Melosh, N. A. Nanostraw-Electroporation System for Highly Efficient Intracellular Delivery and Transfection. ACS Nano 2013, 7 (5), $4351-4358$ 
(37) Xu, A. M.; Aalipour, A.; Leal-Ortiz, S.; Mekhdjian, A. H.; Xie, X.; Dunn, A. R.; Garner, C. C.; Melosh, N. A. Quantification of nanowire penetration into living cells. Nat. Commun. 2014, 5, 3613.

(38) VanDersarl, J. J.; Xu, A. M.; Melosh, N. A. Nanostraws for Direct Fluidic Intracellular Access. Nano Lett. 2012, 12 (8), 38813886.

(39) De Angelis, F.; Malerba, M.; Patrini, M.; Miele, E.; Das, G.; Toma, A.; Zaccaria, R. P.; Di Fabrizio, E. 3D Hollow Nanostructures as Building Blocks for Multifunctional Plasmonics. Nano Lett. 2013, 13 (8), 3553-3558.

(40) Caprettini, V.; Huang, J.-a.; Moia, F.; Jacassi, A.; Gonano, C. A.; Maccaferri, N.; Capozza, R.; Dipalo, M.; De Angelis, F. Enhanced Raman investigation of cell membrane and intracellular compounds by 3D plasmonic nanoelectrode arrays. Adv. Sci. 2018, 5, 1800560.

(41) Goddard, G.; Brown, L. O.; Habbersett, R.; Brady, C. I.; Martin, J. C.; Graves, S. W.; Freyer, J. P.; Doorn, S. K. HighResolution Spectral Analysis of Individual SERS-Active Nanoparticles in Flow. J. Am. Chem. Soc. 2010, 132 (17), 6081-6090.

(42) Hill, E. K.; de Mello, A. J. Single-molecule detection using confocal fluorescence detection: Assessment of optical probe volumes. Analyst 2000, 125 (6), 1033-1036.

(43) Cecchini, M. P.; Stapountzi, M. A.; McComb, D. W.; Albrecht, T.; Edel, J. B. Flow-Based Autocorrelation Studies for the Detection and Investigation of Single-Particle Surface-Enhanced Resonance Raman Spectroscopic Events. Anal. Chem. 2011, 83 (4), 1418-1424.

(44) Barbara, A.; Dubois, F.; Ibanez, A.; Eng, L. M.; Quemerais, P. SERS Correlation Spectroscopy of Silver Aggregates in Colloidal Suspension: Quantitative Sizing Down to a Single Nanoparticle. J. Phys. Chem. C 2014, 118 (31), 17922-17931.

(45) Liu, L.; Kong, J.; Xie, X.; Wu, H.; Ye, X.; Zhao, Z.; Wang, L.; Liu, Q. Gold nanorod translocation through a solid-state nanopore. Chin. Sci. Bull. 2014, 59 (7), 598-605.

(46) Angeli, E.; Volpe, A.; Fanzio, P.; Repetto, L.; Firpo, G.; Guida, P.; Lo Savio, R.; Wanunu, M.; Valbusa, U. Simultaneous ElectroOptical Tracking for Nanoparticle Recognition and Counting. Nano Lett. 2015, 15 (9), 5696-5701.

(47) Cecchini, M. P.; Wiener, A.; Turek, V. A.; Chon, H.; Lee, S.; Ivanov, A. P.; McComb, D. W.; Choo, J.; Albrecht, T.; Maier, S. A.; Edel, J. B. Rapid Ultrasensitive Single Particle Surface-Enhanced Raman Spectroscopy Using Metallic Nanopores. Nano Lett. 2013, 13 (10), 4602-4609.

(48) Qiu, Y. H.; Siwy, Z. Probing charges on solid-liquid interfaces with the resistive-pulse technique. Nanoscale 2017, 9 (36), 1352713537.

(49) Keij, J. F.; Van Rotterdam, A.; Groenewegen, A. C.; Stokdijk, W.; Visser, J. W. M. COINCIDENCE IN HIGH-SPEED FLOWCYTOMETRY - MODELS AND MEASUREMENTS. Cytometry 1991, 12 (5), 398-404.

(50) Chen, P.; Mitsui, T.; Farmer, D. B.; Golovchenko, J.; Gordon, R. G.; Branton, D. Atomic layer deposition to fine-tune the surface properties and diameters of fabricated nanopores. Nano Lett. 2004, 4 (7), 1333-1337.

(51) Kosmulski, M. The $\mathrm{pH}$-dependent surface charging and the points of zero charge. J. Colloid Interface Sci. 2002, 253 (1), 77-87.

(52) Schoch, R. B.; Han, J.; Renaud, P. Transport phenomena in nanofluidics. Rev. Mod. Phys. 2008, 80 (3), 839-883.

(53) Firnkes, M.; Pedone, D.; Knezevic, J.; Doeblinger, M.; Rant, U. Electrically Facilitated Translocations of Proteins through Silicon Nitride Nanopores: Conjoint and Competitive Action of Diffusion, Electrophoresis, and Electroosmosis. Nano Lett. 2010, 10 (6), 21622167.

(54) Saulis, G.; Saule, R. Size of the pores created by an electric pulse: Microsecond vs millisecond pulses. Biochim. Biophys. Acta, Biomembr. 2012, 1818 (12), 3032-3039.

(55) Huefner, A.; Kuan, W. L.; Muller, K. H.; Skepper, J. N.; Barker, R. A.; Mahajan, S. Characterization and Visualization of Vesicles in the Endo-Lysosomal Pathway with Surface-Enhanced Raman Spectroscopy and Chemometrics. ACS Nano 2016, 10 (1), 307-316.
(56) Huefner, A.; Kuan, W. L.; Barker, R. A.; Mahajan, S. Intracellular SERS Nanoprobes For Distinction Of Different Neuronal Cell Types. Nano Lett. 2013, 13 (6), 2463-2470.

(57) Sirimuthu, N. M. S.; Syme, C. D.; Cooper, J. M. Investigation of the stability of labelled nanoparticles for SE(R)RS measurements in cells. Chem. Commun. 2011, 47 (14), 4099-4101.

(58) Liu, M.; Li, Q.; Liang, L.; Li, J.; Wang, K.; Li, J.; Lv, M.; Chen, N.; Song, H.; Lee, J.; Shi, J.; Wang, L.; Lal, R.; Fan, C. Real-time visualization of clustering and intracellular transport of gold nanoparticles by correlative imaging. Nat. Commun. 2017, 8, 15646.

(59) Demiryurek, Y.; Nickaeen, M.; Zheng, M. D.; Yu, M.; Zahn, J. D.; Shreiber, D. I.; Lin, H.; Shan, J. W. Transport, resealing, and reporation dynamics of two-pulse electroporation-mediated molecular delivery. Biochim. Biophys. Acta, Biomembr. 2015, 1848 (8), 17061714.

(60) Teissie, J.; Golzio, M.; Rols, M. P. Mechanisms of cell membrane electropermeabilization: A minireview of our present (lack of ?) knowledge. Biochim. Biophys. Acta, Gen. Subj. 2005, 1724 (3), 270-280.

(61) Cao, Y.; Hjort, M.; Chen, H.; Birey, F.; Leal-Ortiz, S. A.; Han, C. M.; Santiago, J. G.; Pasca, S. P.; Wu, J. C.; Melosh, N. A. Nondestructive nanostraw intracellular sampling for longitudinal cell monitoring. Proc. Natl. Acad. Sci. U. S. A. 2017, 114 (10), E1866E1874.

(62) He, G.; Yang, C.; Hang, T.; Liu, D.; Chen, H.-J.; Zhang, A.-H.; Lin, D.-A.; Wu, J.; Yang, B.-R.; Xie, X. Hollow NanoneedleElectroporation System to Extract Intracellular Protein Repetitively and Nondestructively. ACS Sens. 2018, 3, 1675.

(63) Boyd, D.; Beckwith, J. POSITIVELY CHARGED AMINOACID RESIDUES CAN ACT AS TOPOGENIC DETERMINANTS IN MEMBRANE-PROTEINS. Proc. Natl. Acad. Sci. U. S. A. 1989, 86 (23), 9446-9450.

(64) Dou, X. M.; Jung, Y. M.; Cao, Z. Q.; Ozaki, Y. Surfaceenhanced Raman scattering of biological molecules on metal colloid II: Effects of aggregation of gold colloid and comparison of effects of $\mathrm{pH}$ of glycine solutions between gold and silver colloids. Appl. Spectrosc. 1999, 53 (11), 1440-1447.

(65) Zakaria, H. M.; Shah, A.; Konieczny, M.; Hoffmann, J. A.; Nijdam, A. J.; Reeves, M. E. Small Molecule- and Amino AcidInduced Aggregation of Gold Nanoparticles. Langmuir 2013, 29 (25), $7661-7673$

(66) Hernandez, B.; Tinacci, L.; Coic, Y.-M.; Chenal, A.; Cohen, R.; Sanchez-Cortes, S.; Ghomi, M. Tryptophan Tight Binding to Gold Nanoparticles Induces Drastic Changes in Indole Ring Raman Markers. J. Phys. Chem. C 2018, 122 (24), 13034-13046.

(67) Etoc, F.; Balloul, E.; Vicario, C.; Normanno, D.; Lisse, D.; Sittner, A.; Piehler, J.; Dahan, M.; Coppey, M. Non-specific interactions govern cytosolic diffusion of nanosized objects in mammalian cells. Nat. Mater. 2018, 17 (8), 740.

(68) Cerea, A.; Caprettini, V.; Bruno, G.; Lovato, L.; Melle, G.; Tantussi, F.; Capozza, R.; Moia, F.; Dipalo, M.; De Angelis, F. Selective intracellular delivery and intracellular recordings combined in MEA biosensors. Lab Chip 2018, 18 (22), 3492-3500.

(69) Bohndiek, S. E.; Wagadarikar, A.; Zavaleta, C. L.; Van de Sompel, D.; Garai, E.; Jokerst, J. V.; Yazdanfar, S.; Gambhir, S. S. A small animal Raman instrument for rapid, wide-area, spectroscopic imaging. Proc. Natl. Acad. Sci. U. S. A. 2013, 110 (30), 12408-12413.

(70) Kang, J. W.; Nguyen, F. T.; Lue, N.; Dasari, R. R.; Heller, D. A. Measuring Uptake Dynamics of Multiple Identifiable Carbon Nanotube Species via High-Speed Confocal Raman Imaging of Live Cells. Nano Lett. 2012, 12 (12), 6170-6174.

(71) Higgins, S. G.; Stevens, M. M. Extracting the contents of living cells. Science 2017, 356 (6336), 379-380.

(72) Santoro, F.; Zhao, W. T.; Joubert, L. M.; Duan, L. T.; Schnitker, J.; van de Burgt, Y.; Lou, H. Y.; Liu, B. F.; Salleo, A.; Cui, L. F.; Cui, Y.; Cui, B. X. Revealing the Cell-Material Interface with Nanometer Resolution by Focused Ion Beam/Scanning Electron Microscopy. ACS Nano 2017, 11 (8), 8320-8328. 
(73) Lanzano, L.; Scipioni, L.; Di Bona, M.; Bianchini, P.; Bizzarri, R.; Cardarelli, F.; Diaspro, A.; Vicidomini, G. Measurement of nanoscale three-dimensional diffusion in the interior of living cells by STED-FCS. Nat. Commun. 2017, 8, 65.

(74) Rakic, A. D.; Djurisic, A. B.; Elazar, J. M.; Majewski, M. L. Optical properties of metallic films for vertical-cavity optoelectronic devices. Appl. Opt. 1998, 37 (22), 5271-5283. 